\title{
Novel Domain-Specific Actions of Amyloid Precursor Protein on Developing Synapses
}

\author{
Takako Morimoto, Ikuroh Ohsawa, Chizuko Takamura, Mariko Ishiguro, Yasuko Nakamura, and \\ Shinichi Kohsaka
}

Department of Neurochemistry, National Institute of Neuroscience, Kodaira, Tokyo 187-8502, Japan

\begin{abstract}
The effect of the secretory form of amyloid precursor protein (sAPP) on synaptic transmission was examined by using developing neuromuscular synapses in Xenopus cell cultures. The frequency of spontaneous postsynaptic currents (SSCs) was reduced by the addition of SAPP, whereas the amplitude of impulse-evoked postsynaptic currents (ESCs) was increased by sAPP. These opposing effects on spontaneous versus evoked release were separated by using the specific domain of APP. The C-terminal fragment of SAPP (CAPP) only reduced SSC frequency and did not affect ESCs. By contrast, the $\mathrm{N}$-terminal fragment of SAPP (NAPP) did not affect SSC fre-
\end{abstract}

quency but did increase ESC amplitude. The reduction of SSC frequency by SAPP appears to be mediated by activation of potassium channels through a cGMP-dependent pathway, whereas the increase of ESC amplitude is mediated by a different pathway involving activation of protein kinase(s). These results suggest the potential role of SAPP as a modulator of synaptic activity by two specific domains.

Key words: amyloid precursor protein; spontaneous synaptic currents; evoked synaptic currents; domain-specific; modulator; synaptic activity; developing synapses
Amyloid precursor protein (APP) is the source of $\beta$ A 4 peptide, which is a major component of the amyloid deposits characterized in Alzheimer's disease (AD) (Selkoe, 1991). Although it has not yet been established, there are increasing data indicating that APP is causally involved in the pathogenesis of AD (Selkoe, 1991; Mattson et al., 1993). For example, mutations in APP have been shown to result in the inherited forms of $\mathrm{AD}$ in several cases (Hardy, 1992). Moreover, the physiological role of APP in the development and modulation of synaptic functions has been emphasized recently. APP is widely expressed in neuronal cells, even in the developing nervous systems (Card et al., 1988; Kang and Muller-Hill, 1990; Moya et al., 1994; Salbaum and Ruddle, 1994; Yamazaki et al., 1995) and is accumulated in the synaptic sites (Schubert et al., 1991). The secretory form of APP (sAPP) is released from membrane-spanning APP by electrical stimulation of neural circuits in hippocampal slices (Nitsch et al., 1993), and sAPP has been shown to stabilize intracellular calcium concentrations in studies on cultured hippocampal cells (Mattson et al., 1993). This effect of sAPP is attributable to the activation of potassium channels through a cGMP-dependent pathway (Furukawa et al., 1996a). Furthermore, modulatory effects of sAPP on synaptic activities have been reported, particularly in the hippocampal slices (Ishida et al., 1997). In this report, sAPP lowered the tetanus frequency to induce long-term depression (LTD) and enhanced long-term potentiation (LTP) induced by high-frequency stimulation. These results suggest involvement of APP in neural activation, but whether APPs enhance or suppress the level of neural activation is still controversial. Here, we

Received June 15, 1998; revised Aug. 24, 1998; accepted Sept. 1, 1998.

This study was supported by a Center of Excellence (COE) grant from the Science and Technology Agency, Japan, and a grant from the Japanese Ministry of Health and Welfare.

Correspondence should be addressed to Dr. S. Kohsaka, Department of Neurochemisty, National Institute of Neuroscience, 4-1-1 Ogawa-higashi, Kodaira, Tokyo 187-8502, Japan.

Copyright (C) 1998 Society for Neuroscience $\quad 0270-6474 / 98 / 189386-08 \$ 05.00 / 0$ examined the effects of sAPP on the synaptic activity of a developing neuromuscular synapse in Xenopus cell cultures. We found that SAPP modulated the synaptic activity by two specific domains. The C-terminal fragment of sAPP (CAPP) reduced the frequency of the spontaneous synaptic currents (SSCs), and did not affect the evoked synaptic currents (ESCs). By contrast, the $\mathrm{N}$-terminal fragment of sAPP (NAPP) did not affect SSCs but did increase ESC amplitude. The reduction of SSC frequency by sAPP appears to be mediated by activation of potassium channels through a cGMP-dependent pathway, whereas the increase of ESC amplitude is mediated by a different pathway involving activation of protein kinase(s). These results provide direct evidence of the acute domain-specific actions of sAPP in modulating the basal synaptic function of developing synapses.

\section{MATERIALS AND METHODS}

Preparation of $S A P P, N$-terminal fragment of $A P P$, and $C$-terminal fragment of $A P P$. SAPP, NAPP, and CAPP (Fig. 1) were prepared as described previously (Ohsawa et al., 1995, 1997). In brief, human APP695 cDNA was subcloned into pAE1 vector, and NAPP cDNA encoding from $\mathrm{Arg}^{16}$ to $\mathrm{Val}^{290}$ of human APP695 was subcloned into pAE3 vector. CAPP cDNA encoding from Glu ${ }^{305}$ to $\mathrm{Thr}^{588}$ of human APP695 was also subcloned. Culture medium was separated from the yeast transfected with these vectors, and the proteins were purified using a heparin Sepharose column, an alkyl-Superose column, and a DE52 column, respectively. The purification of sAPP, CAPP, and NAPP was monitored by SDS-PAGE and analyzed by silver staining or Western blotting with monoclonal antibody $22 \mathrm{C} 11$ and Alz-90 (Boehringer Mannheim, Tokyo, Japan). NAPP contained cysteine-rich motifs and an acidic region, which gave a higher charge than that of CAPP. Predicted isoelectric points for SAPP, NAPP, and CAPP were 4.5, 4.0, and 5.5, respectively.

Cell culture. Xenopus spinal motoneurons and myotomal myocytes were prepared from stage 20-22 embryos and cultured according to previously reported methods (Spitzer and Lamborghini, 1976). The dissociated cells were plated on coverslips in the culture medium consisting of $50 \%(\mathrm{v} / \mathrm{v})$ Leibovitz's medium (Life Technologies, Gaithersburg, MD), $1 \%(\mathrm{v} / \mathrm{v})$ fetal calf serum (Life Technologies), and $49 \%(\mathrm{v} / \mathrm{v})$ Ringer's solution $(115 \mathrm{~mm} \mathrm{NaCl}, 2 \mathrm{~mm} \mathrm{CaCl} 2,2.5 \mathrm{~mm} \mathrm{KCl}$, and $10 \mathrm{~mm}$ 


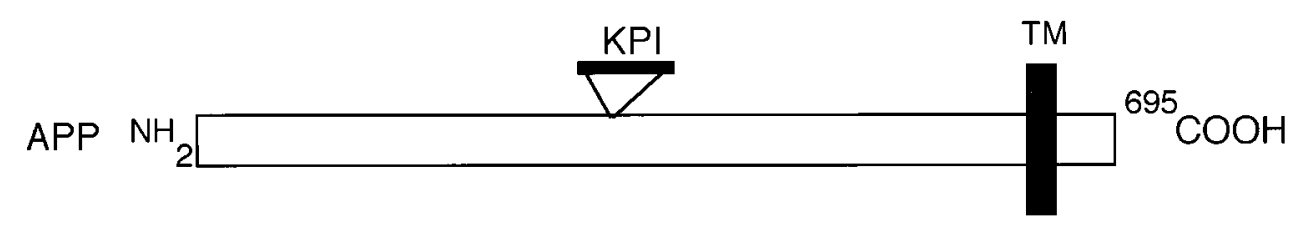

SAPP

$1 8 \longdiv { \square }$

NAPP

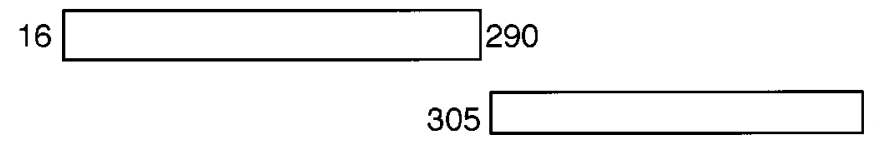

CAPP

612 588

Figure 1. Structure of APP, sAPP, NAPP, and CAPP. The numbers represent the amino acid sequences. The position of the transmembrane domain $(T M)$ and the Knitz-type protease inhibitor domain $(K P I)$ are indicated.

HEPES, $\mathrm{pH}$ 7.3). The cultures were maintained for $1 \mathrm{~d}$ at room temperature $\left(24^{\circ} \mathrm{C}\right)$ and used for the electrophysiological experiments.

Electrophysiology. Gigaohm-seal whole-cell recording from muscle cells followed those described previously (Hamill et al., 1981). All recordings were performed at room temperature $\left(24^{\circ} \mathrm{C}\right)$. The solution inside the whole-cell recording pipette contained $150 \mathrm{mM} \mathrm{KCl}, 1 \mathrm{mM}$ $\mathrm{NaCl}, 1 \mathrm{~mm} \mathrm{MgCl}$, and $10 \mathrm{~mm}$ HEPES, $\mathrm{pH}$ 7.2. The membrane currents were monitored with a patch-clamp amplifier (Nihon Koden, Tokyo, Japan). The recorded currents were filtered at $5 \mathrm{~Hz}$, stored on a DAT recording tape for later playback, and analyzed with the SCAN program (kindly provided by Dr. J. Dempster, Strathclyde University, Glascow, UK). Chemicals tested were dissolved in the culture medium. SSCs were recorded, and the frequency of the SSCs was determined by counting the number of events per minute. We have followed the procedure described previously to analyze fluctuations of ESC amplitude, assuming that evoked acetylcholine (ACh) secretion at these synapses is quantal in nature (Bekkers and Stevens, 1990; Malinow and Tsien, 1990; Lo and Poo, 1994). The mean ESC amplitude $(m)$ and its variance $(v)$ were measured before and after the addition of sAPP. The distribution of ESC amplitude was assumed to be a binomial distribution modified to take into account the variability of the quantal size. Thus, $m=a N p$ and $v=$ $a^{2} N p(1-p)+a^{2} N p c_{\mathrm{m}}{ }^{2}$, where $a$ is the average quantal size, $N$ is the total number of available quanta, $p$ is the release probability, and $c_{\mathrm{m}}$ is the coefficient of variation of the quanta. Coefficient of variation $(\mathrm{CV})$ is defined as $v^{1 / 2} / m$. The square of CV, $C V^{2}=\left(1+c_{\mathrm{m}}{ }^{2}-p\right) /(N p)$, is a function independent of quantal size $a$. If an increase of ESC amplitude by SAPP is attributable simply to a change in postsynaptic sensitivity, then $\mathrm{CV}^{2}$ will not change after the addition of sAPP, and the ratio of $\mathrm{CV}^{2}$ before and after the addition of sAPP should remain 1. Substantial deviation from this prediction suggests involvement of presynaptic modulation. Other statistical analysis was done by ANOVA.

Intracellular calcium monitoring. The intracellular calcium concentration $\left(\left[\mathrm{Ca}^{2+}\right]_{\mathrm{i}}\right)$ was monitored with the calcium indicator, fura-2 AM (Dojindo, Kumamoto, Japan) by the modified method described previously (Morimoto et al., 1998a). In brief, Xenopus neuromuscular cultures were incubated with Ringer's solution supplemented with $10 \mu \mathrm{M}$ fura-2 $\mathrm{AM}$ for $1 \mathrm{hr}$ at room temperature. After washing twice with Ringer's solution, cells were exposed at 340 and $360 \mathrm{~nm}$ excitation beams, and fluorescent images were recorded with a highly sensitive intensifier target camera (Hamamatsu Photonics, Hamamatsu, Japan). Experiments were performed at room temperature. After image acquisition, data were expressed as ratios of fluorescence intensity at 340 and $360 \mathrm{~nm}$ and analyzed by Argus-50 (Hamamatsu Photonics).

Chemicals. 8-br-cGMP and Rp-8-br-cGMPs were purchased from Biomol Research Laboratories (Plymouth Meeting, PA). All other chemicals were of analytical grade.

\section{RESULTS}

\section{Effect of sAPP on spontaneous synaptic currents}

Modulatory effects of sAPP on synaptic transmission were examined at developing neuromuscular synapses in 1-d-old Xenopus nerve-muscle cultures. In the first set of experiments, SSCs were monitored from innervated myocytes by whole-cell voltage-clamp recording. These currents are caused by spontaneous pulsatile ACh release from nerve terminals, because they are abolished by bath application of D-tubocurarine and are unaffected by tetro- dotoxin, which blocks action potentials in neurons (Xie and Poo, 1986). As shown in Figure $2 B$, bath application of sAPP (20 nm) reduced the frequency of SSCs within $5 \mathrm{~min}$. Figure $2 C$ shows the mean frequency during the first $15 \mathrm{~min}$ after the addition of culture medium (Fig. $2 C$, cont) or sAPP (5 nM, $20 \mathrm{nM})$ normalized to the mean frequency for $5 \mathrm{~min}$ before the addition. The reduction of the SSC frequency was dependent on the dose of SAPP added to the medium, and at $20 \mathrm{~nm}$ sAPP the frequency was significantly reduced ( $p<0.05$, ANOVA). Further analysis of SSC properties was performed to investigate whether the effect of sAPP was on the presynaptic or postsynaptic mechanisms. We found that the mean amplitude was not significantly affected by the addition of sAPP (Fig. 2D). Furthermore, there was no detectable change in the distribution of SSC amplitude (Fig. $3 A$ ), rise time (Fig. $3 B$ ), or half-decay time (Fig. $3 C$ ) after the addition of sAPP ( $p>0.05$, ANOVA). These results suggest that the ACh sensitivity of the postsynaptic sites has not been affected by the addition of sAPP. Therefore, it is most likely that the primary action of sAPP on these synapses is a modulation of the presynaptic ACh secretion mechanism.

\section{Effect of sAPP on evoked synaptic currents}

To examine the effect of sAPP on the impulse-evoked synaptic response, the membrane currents of innervated myocytes were recorded in response to extracellular suprathreshold stimulation of the neuronal soma at a frequency lower than $0.1 \mathrm{~Hz}$. In Figure $4 A$, the neuronal soma was stimulated at the points marked by a small line before and after the addition of sAPP solution (at the final bath concentration of $20 \mathrm{~nm}$ ). The amplitude of ESCs was increased after the addition of sAPP. Figure $4 B$ shows the timedependent change of the mean ESC amplitude normalized to the mean ESC amplitude before treatment. The mean ESC amplitude was calculated from the amplitude of ESCs evoked by 5-10 test stimuli. A gradual decline in the mean amplitude of ESCs was usually observed in the recordings from the control synapses with culture medium, as reported previously (Lohof et al., 1993; Lo and Poo, 1994), presumably as a result of slow synaptic depression caused by the test stimulation. The mean amplitude of ESCs was increased $5 \mathrm{~min}$ after the addition of sAPP to the bath. The increase in ESC amplitude gradually declined after $15 \mathrm{~min}$, as shown in Figure $4 B$. The mean ESC amplitude during the 5-15 min after the addition of sAPP normalized to the mean amplitude before the addition was $1.20 \pm 0.13(n=21)$ (Fig. $5 B)$ and was significantly different from the value of the control synapses to which culture medium had been added $(0.85 \pm 0.06, n=12, p<$ 0.05, ANOVA). To determine whether the observed effect of sAPP on ESC amplitude was attributable solely to a change in quantal size, for example, resulting from a change in sensitivity of 
A

Figure 2. Effect of sAPP on the SSCs. A, B, The continuous trace shows the membrane currents recorded from innervated myocytes before and after application of culture medium $(A)$ and sAPP (20 nM) (B). Arrows indicate the point at which the solution was added. The insets below depict the average traces of SSCs during indicated times at higher time resolution. Calibration: fast traces, $35 \mathrm{msec}, 0.35 \mathrm{nA}$; slow traces, 3 min, $0.5 \mathrm{nA}$. $C$, The frequency of SSCs during the 15 min after application of culture medium (cont; $n=8)$ and sAPP (5 nM, $n=5 ; 20 \mathrm{nM}, n=$ 14) was normalized to the frequency of SSCs for the $5 \mathrm{~min}$ before treatment. The data points represent means \pm SEM. Values marked with an asterisk are significantly different from the control value ( $p<0.05$, ANOVA). $D$, The mean amplitude of SSCs during the 15 min after application of culture medium (cont) and SAPP was normalized to the mean amplitude for the 5 min before treatment. The data points represent means \pm SEM.

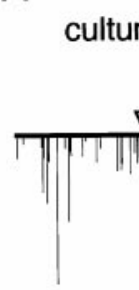

culture medium

$\nabla$

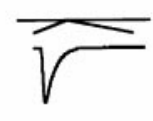

C

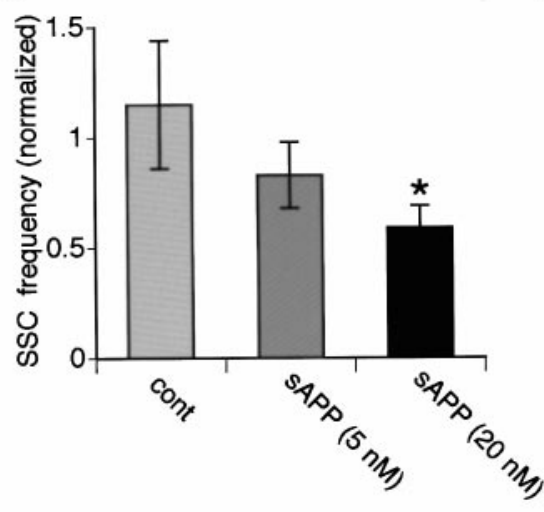

B

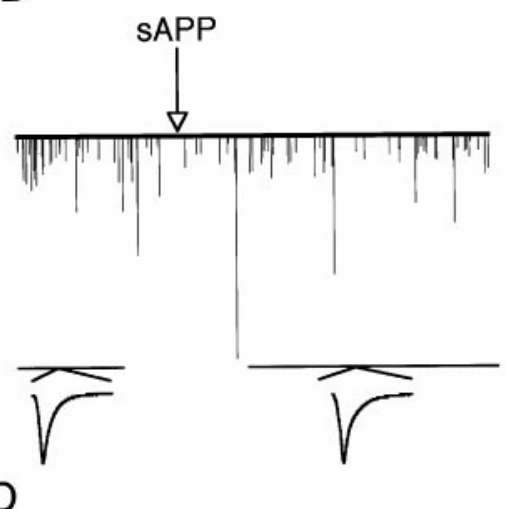

postsynaptic ACh receptors, or modulation of presynaptic release mechanisms, the fluctuation of the ESC amplitude was analyzed. As described in the previous report (Lo and Poo, 1994), the CV of ESC amplitudes should theoretically remain unchanged after the addition of SAPP if the effect of sAPP is caused by a change in quantal size. We measured the CV of ESC amplitudes before $\left(C V_{\mathrm{b}}\right)$ and after $\left(C V_{\mathrm{a}}\right)$ the addition of sAPP, and as shown in Figure $4 C$, the ratios of $\mathrm{CV}$ squared $\left(C V_{\mathrm{a}}^{2} / C V_{\mathrm{b}}{ }^{2}\right)$ deviated significantly from unity (broken line) in the 17 experiments in which a sufficient number of ESC events were collected for the analysis. Thus, the increase in ESC amplitude induced by sAPP is unlikely to be accounted for by a simple increase in quantal size. An increase in the number of available quanta and the probability of quantal release are likely to be involved.

\section{Domain-specific effects of sAPP}

Several domains of APP have been shown to exhibit the specific distinct function (Ninomiya et al., 1993; Furukawa et al., 1996b). To examine the effective domain for two effects of sAPP, i.e., reduction of SSC frequency and increase of ESC amplitude, we prepared two sAPP fragments: NAPP, which includes residue 16-290 of APP695, and CAPP, which includes residues 305-588 of APP695 (Fig. 1). Because sAPP was divided at the insertion point of the Knitz-type protease inhibitor (KPI), each fragment could maintain the secondary structure, which is possibly important to exert the biological activity. SSCs and ESCs were examined before and after the addition of either NAPP (40 nM) or CAPP (40 nM) into the bath. The frequency of the SSCs and the mean amplitude of the ESCs for 15 min after the addition of the factor were normalized to mean values before their addition (Fig. $5 A, B)$. No reduction in SSC frequency was observed as a result of the addition of NAPP $(n=13)$. Surprisingly, an increase of ESC amplitude was detected by the addition of NAPP. NAPP increased ESC amplitude to $1.20 \pm 0.11$-fold (SEM, $n=12$ ) of the value before the addition of NAPP, and the effect was significant $(p<0.01$, ANOVA). The effect of NAPP was persistent, and ESC amplitude remains elevated to $1.22 \pm 0.31$-fold of the value before the addition of NAPP at 25 min after the addition of NAPP. By contrast, the addition of CAPP significantly reduced the frequency of the SSCs (Fig. $5 A)(n=18 ; p<0.05$, ANOVA), with no effect on the mean amplitude of SSCs (data not shown). On the other hand, the mean amplitude of ESCs was not affected by CAPP (Fig. $5 B$ ). These results suggest that two distinct effects of sAPP on synaptic activity, reduction of SSC frequency and increase of ESC amplitude, could be induced by the different domains of sAPP, i.e., by the $\mathrm{C}$ - and the $\mathrm{N}$-terminal domains, respectively.

\section{Mechanisms underlying the two domain-specific effects of sAPP}

It has been reported that $\mathrm{SAPP}$ can activate $\mathrm{K}^{+}$channels through the cGMP-dependent pathway, resulting in a reduction of the cytosolic concentration of calcium ions $\left(\left[\mathrm{Ca}^{2+}\right]_{i}\right)$ in cultured hippocampal neurons (Furukawa et al., 1996a). Such modulation of neural excitability was shown to depend on the C-terminal fragment of sAPP (Furukawa et al., 1996b). Because cytosolic $\mathrm{Ca}^{2+}$ is known to influence the frequency of spontaneous quantal secretion (Katz and Miledi, 1969), the reduction of frequency of SSCs by sAPP may be mediated by the same mechanism. Thus, we first examined the effect of sAPP on $\left[\mathrm{Ca}^{2+}\right]_{i}$ of the cultured cells. Figure $6 A$ shows the change of $\left[\mathrm{Ca}^{2+}\right]_{i}$ induced by sAPP. $\left[\mathrm{Ca}^{2+}\right]_{\mathrm{i}}$ was reduced by the addition of sAPP in neurons, but there was no change in $\left[\mathrm{Ca}^{2+}\right]_{i}$ in muscle cells. CAPP also reduced $\left[\mathrm{Ca}^{2+}\right]_{i}$ in neurons (data not shown). Next, we have 
A

B
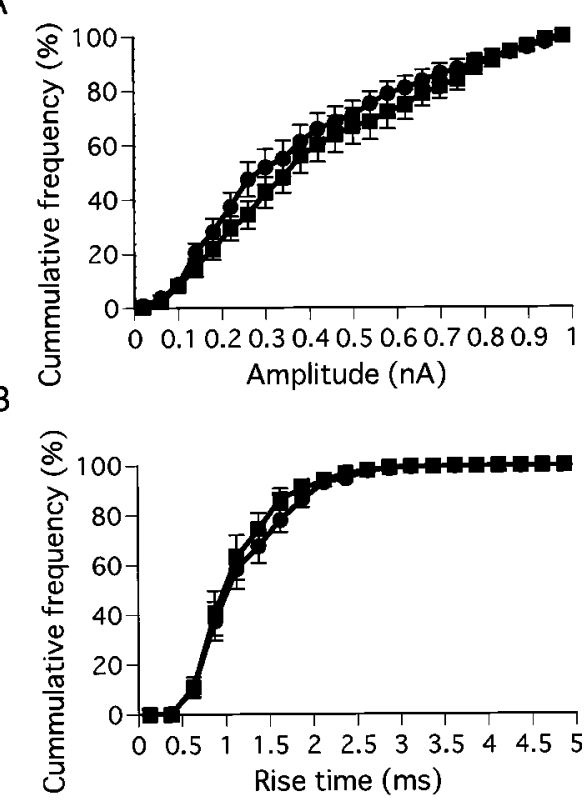

C

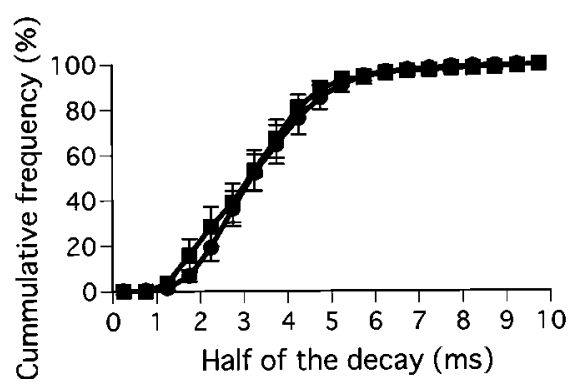

Figure 3. Absence of any changes in the properties of SSCs. The amplitude $(A)$, rise time $(B)$, and half-decay time $(C)$ distributions of SSCs before (ם) and after $(\bullet)$ APP treatment were compared. Cumulative frequency refers to the fraction of total events with values smaller than a given value. The data points represent means $\pm \operatorname{SEM}(n=14)$. All data points are not significantly different between before and after APP treatment $(p>0.05$, ANOVA).

examined the effects of a $\mathrm{K}^{+}$channel activator and cGMP on the SSC frequency (Fig. 6B) and ESC amplitude (Fig. 6C). As shown in Figure $6 B$, the $\mathrm{K}^{+}$channel activator diazoxide could mimic the effect of sAPP on SSC frequency. Bath application of diazoxide at a final concentration of $100 \mu \mathrm{M}$ significantly reduced SSC frequency ( $n=7 ; p<0.01$, ANOVA). Furthermore, 8-br-cGMP, a membrane permeable analog of cGMP, mimics the effect of sAPP on SSC frequency. Bath application of 8-br-cGMP at a final concentration of 600-700 $\mu \mathrm{M}$ significantly reduced SSC frequency ( $n=7 ; p<0.05$, ANOVA). However, both diazoxide and 8-br-cGMP did not alter ESC amplitude (Fig. 6C). These results suggested that the cGMP-dependent mechanism is sufficient in reducing the SSC frequency. That cGMP-dependent activity is necessary for the action of sAPP on the SSC frequency is tested further by the use of a specific competitor of cGMP, Rp-8-brcGMP (RGS). Addition of RGS (30 $\mu \mathrm{M})$ by itself had no effect on either SSC frequency (Fig. 6B) or ESC amplitude (Fig. 6C). However, the same RGS addition inhibited sAPP-induced reduction of SSC frequency (Fig. $6 B$ ), without affecting sAPP-induced increase of ESC amplitude (Fig. 6C). These results strongly suggested that reduction of SSC frequency by sAPP or CAPP was caused by a cGMP-dependent pathway and that the increase of
A
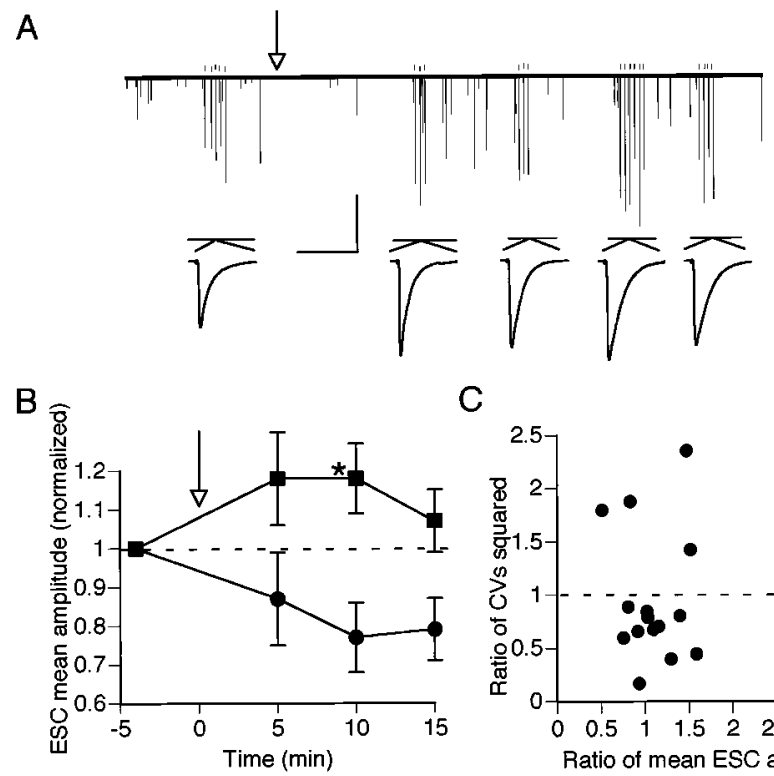

C

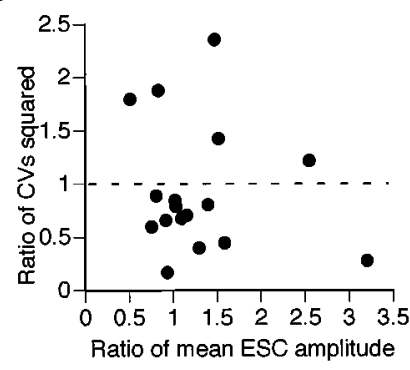

Figure 4. Effect of sAPP on the ESCs. A, The continuous trace shows the membrane current recorded from the innervated myocytes before and after application of SAPP. An arrow indicates the point at which sAPP was added. ESCs were elicited at a frequency below $0.1 \mathrm{~Hz}$ at the times marked with short vertical line. The insets below depict the average ESC events at higher time resolution. Calibration: fast traces, $40 \mathrm{msec}, 1.8 \mathrm{nA}$; slow traces, 2 min, $1.5 \mathrm{nA}$. $B$, Changes in mean amplitude after application of culture medium $(\bullet)$ or sAPP $(\mathbf{\square})$. The mean amplitude of ESCs evoked by $5-10$ test stimuli observed at 5 min intervals after application of the medium was normalized to the mean amplitude before application. The data points represent means \pm SEM (culture medium, $n=3-12$; sAPP, $n=7-21)$. An arrow indicates the point at which the medium was added. Value marked with an asterisk is significantly different from the control value ( $p<0.05$, ANOVA). $C$, Analysis of ESC amplitude fluctuations for sAPP-treated synapses $(n=17)$. The ratio of CVs squared before and after the addition of SAPP has been plotted versus the ratio of the mean ESC amplitude before and after sAPP. Each point represents data from one experiment. The broken line represents the theoretical prediction for pure postsynaptic changes in synaptic responses.

ESC amplitude by sAPP or NAPP was mediated by the different mechanism.

The mechanism for the increase of ESC amplitude by NAPP was tested further. We have reported recently that SAPP activates phospholipase $\mathrm{C} \gamma$ and protein kinase $\mathrm{C}$ (PKC) in rat neocortical neurons (Ishiguro et al., 1998). Because activation of PKC is known to affect synaptic transmission at many synapses (Shapira et al., 1987), we have tested the effect of NAPP in the presence of staurosporine, a broad-spectrum inhibitor of protein kinases including PKC. An example of the experiments is depicted in Figure $7 A, B$. Staurosporine (at the final concentration of $100 \mathrm{~nm}$ ) was added to the bath after the onset of whole-cell recording, and the mean ESC amplitude was determined 10 min after the addition of drug. NAPP was then added to the bath, and the mean ESC amplitude was determined $5 \mathrm{~min}$ after the addition of NAPP. As shown in Figure $7 C$, treatment of staurosporine by itself did not affect ESC amplitude in the time scale of these experiments. In the absence of staurosporine, NAPP significantly increased ESC amplitude (Fig. 7A,C). Pretreatment of staurosporine abolished the increase of ESC amplitude by NAPP (Fig. 7B,C). These results suggest that NAPP increases the ESC amplitude through the activation of protein kinase(s). Figure $7 D$ showed the effect of CAPP on SSC frequency in the presence of $100 \mathrm{~nm}$ 


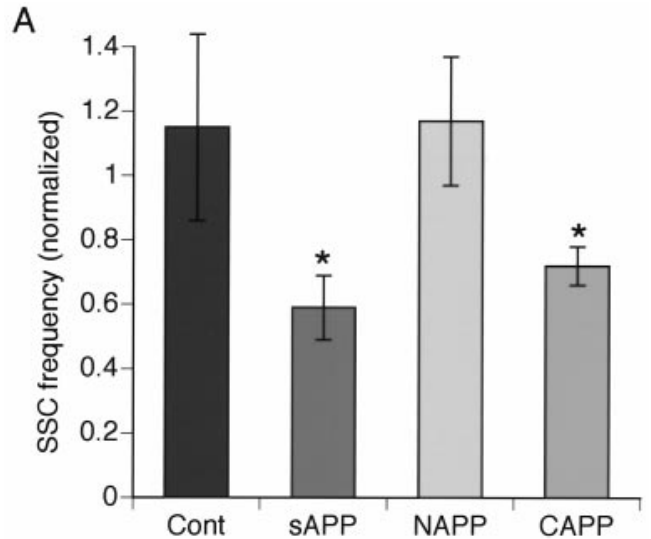

B

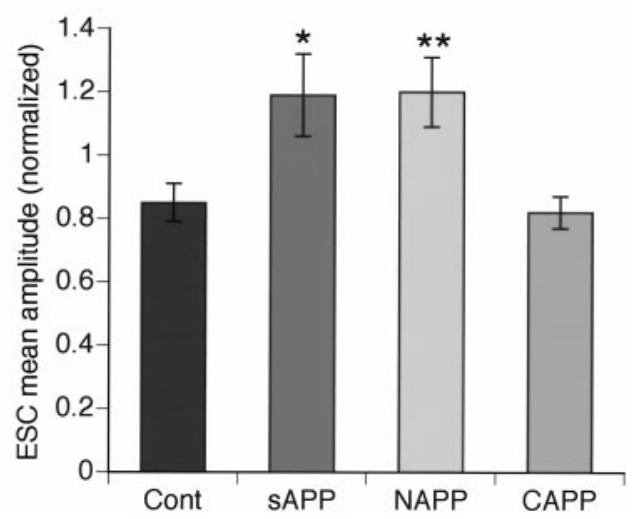

Figure 5. Effect of the N- and C-terminal domain of sAPP (NAPP and CAPP) on SSC frequency and ESC amplitude. A, Averages of the normalized frequency of SSCs after application of culture medium (Cont, $n=8), \operatorname{sAPP}(n=14), N A P P(n=13)$, and $C A P P(n=18)$. The data points represent means \pm SEM. Values marked with an asterisk are significantly different from the control value ( $p<0.05$, ANOVA). $B$, Averages of the normalized mean amplitudes of ESCs after application of culture medium (Cont, $n=12), \operatorname{sAPP}(n=21), N A P P(n=7)$, and CAPP $(n=5)$. The data points represent means \pm SEM. Values marked with one asterisk and two asterisks are significantly different from the control value $\left({ }^{*} p<0.05 ;{ }^{* *} p<0.01\right.$, ANOVA).

staurosporine. Even in the presence of staurosporine, CAPP significantly reduced SSC frequency, suggesting that the reduction of SSC frequency by CAPP was not caused by activation of protein kinase(s).

\section{DISCUSSION}

In this study, we observed two distinct and opposite effects of sAPP on the spontaneous and evoked synaptic activity: a reduction of SSC frequency and an increase in ESC amplitude. Several lines of evidence suggested that these effects were predominantly a result of changes in presynaptic ACh release, instead of changes in postsynaptic ACh response. First, the distribution of SSC amplitude and the time course remained the same after the addition of SAPP, indicating no significant alteration of subsynaptic ACh sensitivity. Second, we observed an increase in the amplitude of ESCs and a reduction in SSC frequency at the same time by the addition of SAPP, inconsistent with a simple change in the postsynaptic sensitivity as an underlying mechanism. Third, analysis of the fluctuation of

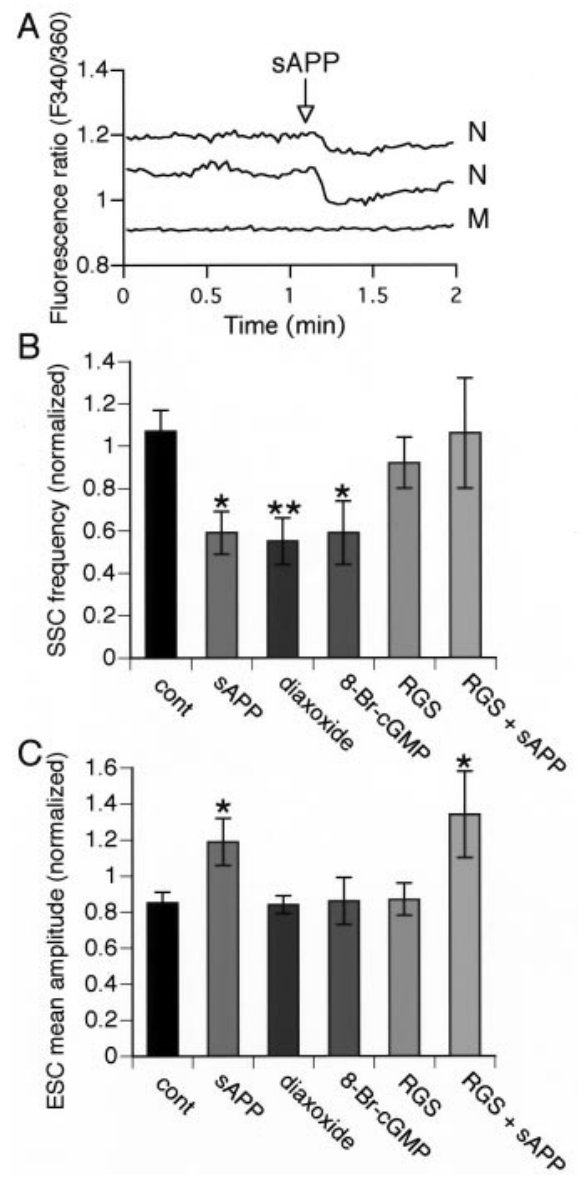

Figure 6. Involvement of $\mathrm{K}^{+}$channel activation and the cGMPdependent pathway in the reduction of SSC frequency. $A$, Examples of $\left[\mathrm{Ca}^{2+}\right]_{\mathrm{i}}$ change in the neuromuscular culture. Fluorescent ratio excised at 340 and $360 \mathrm{~nm}$ was measured. sAPP (20 nM) was added at the indicated time. Reduction of $\left[\mathrm{Ca}^{2+}\right]_{\mathrm{i}}$ was detected in neurons. $N$, Neural cell; $M$, muscle cell. $B$, Effect on SSC frequency of sAPP (20 nM, $n=14)$, diazoxide (100 $\mu \mathrm{M}, n=7)$, 8-br-cGMP (500-700 $\mu \mathrm{M}, n=7)$, RGS (30 $\mu \mathrm{M}, n=5)$, and RGS $(30 \mu \mathrm{M})+\operatorname{sAPP}(20 \mathrm{nM})(n=6)$. SSC frequency for the 15 min after the addition of the chemicals was normalized to the frequency before their addition. The data points represent means \pm SEM. Values marked with one asterisk and two asterisks are significantly reduced compared with the control synapses, to which culture medium was added (cont, $n=5)\left({ }^{*} p<0.05 ; * * p<0.01\right.$, ANOVA). $C$, Effect on ESC amplitude of sAPP (20 nM, $n=21)$, diazoxide $(n=4)$, 8-br-cGMP $(n=$ $7)$, RGS $(n=9)$, and RGS + sAPP $(n=7)$. The data points represent means \pm SEM. Diazoxide, 8-br-cGMP, and RGS did not affect ESC amplitude compared with the control synapses to which culture medium was added (cont, $n=12$ ). Even in the presence of RGS, sAPP significantly increased ESC amplitude $\left({ }^{*} p<0.05\right.$, compared with the control; $p<0.07$, compared with the value of RGS addition; ANOVA).

ESC amplitude further supports the involvement of presynaptic effect.

It is quite surprising that those two effects of sAPP are accounted for by the action of different domains of sAPP and are mediated by the different intracellular mechanisms. The reduction of SSC frequency by SAPP, whose activity resides in CAPP, was mediated by a cGMP-dependent pathway. This possibility was indicated by the finding that an activator of cGMP-dependent pathway, 8-br-cGMP, could mimic the effect of sAPP on SSC frequency and that an inhibitor of the cGMPdependent pathway, RGS, could inhibit it (Fig. 6). These results are consistent with the previous reports that SAPP 


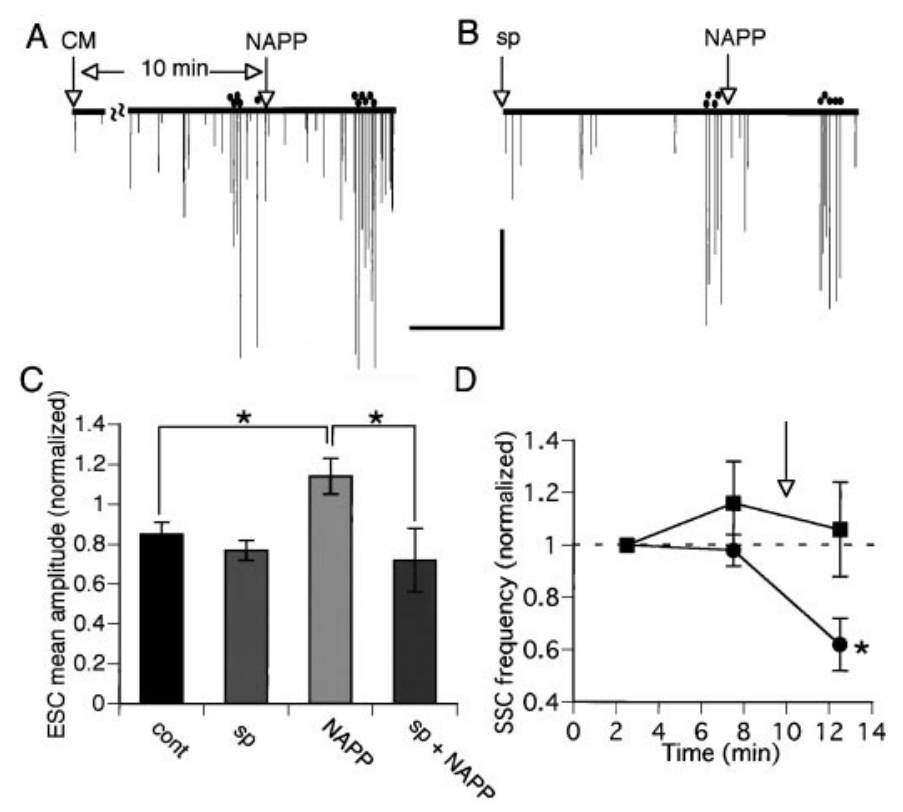

Figure 7. Mechanisms of the increase of ESC amplitude by NAPP. $A, B$, The continuous trace depicts the membrane current recorded from the innervated myocytes before and after application of NAPP in the absence $(A)$ or presence $(B)$ of staurosporine $(s p, 100 \mathrm{~nm})$. Neurons were stimulated at the times indicated $(\bullet), 10 \mathrm{~min}$ after application of culture medium $(A, C M)$ and staurosporine $(B)$, and 5 min after application of NAPP. Calibration: $5 \mathrm{~min}, 400 \mathrm{nA}$. $C$, Average of the mean ESC amplitude at $15 \mathrm{~min}$ after the start of recording, normalized to the value $10 \mathrm{~min}$ after the start of recording. Staurosporine was added at the beginning of recording, and NAPP was added $10 \mathrm{~min}$ after the start of recording, as in $A$ and $B$. Culture medium was added instead of the chemicals in each of the control experiments. NAPP also increased the ESC amplitude with this protocol $\left[n=7,{ }^{*} p<0.05\right.$, ANOVA, compared with control $(n=$ 12)]. Pretreatment with staurosporine significantly inhibited the effect of NAPP on the increase of ESC amplitude ( $n=3,{ }^{*} p<0.05$, ANOVA). The data points represent means \pm SEM. $D$, Reduction of SSC frequency by CAPP in the presence of staurosporine. Staurosporine was added at the beginning of the recording. The arrow indicates the point at which either culture medium $(\boldsymbol{\square}, n=5)$ or $\operatorname{CAPP}(\boldsymbol{\bullet}, n=8)$ was added. SSC frequency was normalized to the frequency for the $5 \mathrm{~min}$ at the beginning of the recording and averaged. CAPP significantly reduced SSC frequency even in the presence of staurosporine $\left({ }^{*} p<0.05\right.$, ANOVA). The data points represent means \pm SEM.

lowers $\left[\mathrm{Ca}^{2+}\right]_{\mathrm{i}}$ by activating potassium channels through a cGMP-dependent pathway in hippocampus cells and that the effective domain resides in the C-terminal (Furukawa et al., $1996 a, b)$. We also found that sAPP reduced $\left[\mathrm{Ca}^{2+}\right]_{i}$ in neurons and that lowering the $\left[\mathrm{Ca}^{2+}\right]_{i}$ by bath application of BAPTA-AM is known to reduce the frequency of SSC at Xenopus developing neuromuscular synapses (Girod et al., 1995).

The activity for increasing the ESC amplitude resides in NAPP. This effect on ESC amplitude is likely to be mediated by the activation of the protein kinase(s), because it was inhibited by staurosporine. That the effect on ESCs is mediated by different intracellular mechanisms from that on SSCs is further supported by the following findings. First, drugs that influence the effect of SAPP on SSC frequency were all ineffective in modifying ESC amplitude. Second, in the presence of staurosporine, CAPP still reduced the SSC frequency. We showed previously that phospholipase $\mathrm{C} \gamma$ and $\mathrm{PKC}$ are activated and growth-associated protein-43 (GAP-43) and myristoylated alanine-rich $\mathrm{C}$ kinase substrate are phosphorylated by
sAPP in rat neocortical neurons (Ishiguro et al., 1998). It has been shown that PKC enhances N- and L-type calcium channel currents in frog sympathetic neurons (Yang and Tsien, 1993). Prolonged action potential-evoked calcium influx into the presynaptic terminal through these calcium channels will lead to increased transmitter release and higher ESC amplitude. GAP-43 and its phosphorylation by PKC were also shown to be involved in the mechanisms of neurotransmitter release (Hens et al., 1995). Further studies are required to clarify the precise mechanism by which the protein kinase mediates the sAPP effect on ESC amplitude. Whether the effect of sAPP on ESC amplitude is caused by a reduction of depression of ESC amplitude or real potentiation of ESC amplitude remains to be elucidated. Depression of ESC attributable to repetitive testing stimulation is well known for developing synapses (Lohof et al., 1993; Lo and Poo, 1994). It is difficult to distinguish between these two possibilities at the present. In either case, the most likely mechanism of the increase in ESC amplitude induced by SAPP and NAPP is an increase in the quantal content, namely, an increase in the number of quanta released by the presynaptic action potential as a result of an increased probability of release or an increased number of available quanta or both.

Development and plasticity in synaptic connections depend on the modulation of synaptic efficacy by electrical and trophic interactions between presynaptic and postsynaptic cells. Modulation of synaptic efficacy by SAPP could also play an important role in the development of the nervous system. Several lines of evidence support this proposal. Expression of APP was abundant and regulated during development in the mammalian embryo. Moya et al. (1994) reported that sAPP increased at the time of synaptogenesis in the hamster visual system and then declined when mature connections were established, suggesting that endogenous SAPP may play an important role in target recognition, synaptic contact, and synaptic maturation in vivo. The APP of Xenopus is highly homologous to human APP, and its expression was increased during the embryonic stages (Okado and Okamoto, 1992). In fact, significant expression of APP mRNA was detected by RT-PCR even at 22 stage Xenopus embryo, from which we prepared the culture (data not shown). So, it is likely that sAPP was secreted endogenously during embryogenesis in vivo. In the previous study (Morimoto et al., 1998b), reduced SSC frequency by overexpression of synaptotagmin in developing spinal neurons resulted in the increased accumulation of synaptic vesicles near the plasma membrane, which reflected a more mature state of presynaptic differentiation. Thus the multiple effects of sAPP on synaptic transmission observed in the present study may reveal a potential function of APP in the development of neuromuscular synapses. On the other hand, it is possible that APP also modulates synaptic activity even in adult neurons because APP is still highly expressed in the adult. In fact, it has been proposed that sAPP contributes to activity-dependent synaptic plasticity such as LTP and LTD and learning and memory. Antibodies against APP have been demonstrated to inhibit the acquisition of a passive avoidance response in the rat (Doyle et al., 1990; Huber et al., 1993). Taken together with the report about an activity-dependent release of sAPP (Nitsch et al., 1993), the effect of NAPP on ESC amplitude resulted in prolonged enhancement of ESC amplitude, suggesting its potential role in LTP.

The intracellular signaling pathways of sAPP have been 
reported, suggesting the presence of receptor-like protein(s) for APP (Furukawa et al., 1996a; Ishiguro et al., 1998). Specific high-affinity binding sites for sAPP on neuronal cells (Ninomiya et al., 1994) also support the existence of receptor-like protein(s) for APP. The presence of two domains in sAPP with different effects on the synaptic properties as shown in the present study suggests the existence of two receptor-like molecules for sAPP. There are many examples of a single ligand having multiple types of receptor and mobilizing various different signals in the cell. Neurotransmitters, such as glutamate and acetylcholine, have at least two types of receptor: an ion-channel type and a G-protein-coupled type (Morita and Katayama, 1984; Sugiyama et al., 1989). There are two kinds of opioid receptors, and they have opposite functions (Crain and Schen, 1992). There are also two kinds of receptors for neurotrophic factors, a low-affinity receptor, p75, and high-affinity Trk receptors (Lewin and Barde, 1996). Regulation of the expression of the receptor-like molecules results in the regulated exhibition of these two effects of sAPP. It is worth noting that some synapses showed only the effect of sAPP on either SSCs or ESCs. Because neurons in this culture are not homogeneous, this may suggest the heterogeneity of those neurons, such as heterogeneous expression of the receptor-like molecules for APP in each neuron. Further identification of the receptor-like molecule(s) for APP will clarify the precise physiological function of APP. In addition to the receptor-like molecules for APP, regulation of APP processing could also lead to either enhancement or reduction of synaptic activity. It has been suggested that APP can be processed either (1) via a nonamyloidogenic pathway in which SAPP is released into the extracellular space or (2) via an alternative pathway that generates $\mathrm{A} \beta$-related peptides. $\mathrm{A} \beta$ peptide was shown to modulate cholinergic synaptic activity. In picomole to nanomole order, A $\beta$ peptide acutely reduced potassium-evoked ACh secretion by cortical and hippocampal brain slices (Kar et al., 1996). Thus, the distinct function of APP as a modulator of synaptic activity might be the key for the switch between physiological and pathological conditions. Enhancement of evoked synaptic activity by NAPP may suggest its neuroprotective role against the reduction of synaptic activity induced by $\mathrm{A} \beta$ peptide.

In conclusion, we have found that sAPP has two novel synaptic modulatory actions, each exerted by a distinct domain in the protein. We have shown further that these actions are mediated by different intracellular mechanisms. Taken together, these results provide direct evidence for a multifunctional role of APP in modulating synaptic functions and suggest a developmental and neuroprotective role of APP.

\section{REFERENCES}

Bekkers JM, Stevens CF (1990) Presynaptic mechanism for long-term potentiation in the hippocampus. Nature 346:724-729.

Card JP, Meade RP, Davis LG (1988) Immunocytochemical localization of the precursor protein for $\beta$-amyloid in the rat central nervous system. Neuron 1:835-846.

Crain SM, Shen K-F (1992) After chronic opioid exposure sensory neurons become supersensitive to the excitatory effects of opioid agonists and antagonists as occurs after acute elevation of GM1 ganglioside. Brain Res 575:13-24.

Doyle E, Bruce MT, Breen KC, Smith DC, Anderton B, Regan CM (1990) Intraventricular infusions of antibodies to amyloid- $\beta$-protein precursor impair the acquisition of a passive avoidance response in the rat. Neurosci Lett 115:97-102.

Furukawa K, Barger SW, Blalock EM, Mattson MP (1996a) Activation of $\mathrm{K}^{+}$channels and suppression of neuronal activity by secreted $\beta$-amyloid precursor protein. Nature 379:74-78.

Furukawa K, Sopher BL, Rydel RE, Begley JG, Pham DG, Martin GM, Fox M, Mattson MP (1996b) Increased activity regulating and neuroprotective efficacy of $\alpha$-secretase-derived secreted amyloid precursor protein conferred by a C-terminal heparin-binding domain. J Neurochem 67:1882-1896.

Girod R, Popov S, Alder J, Zheng JQ, Poo M-m (1995) Spontaneous quantal transmitter secretion from myocytes and fibroblasts: comparison with neuronal secretion. J Neurosci 15:2826-2838.

Hamill OP, Marty A, Neher E, Sakmann B, Sigworth FJ (1981) Improved patch-clamp techniques for high-resolution current recording from cells and cell-free membrane patches. Pflügers Arch 391:85-100.

Hardy J (1992) Framing $\beta$-amyloid. Nat Genet 1:233-234.

Hens JJH, Wit MD, Boomsma F, Mercken M, Oestreicher AB, Gispen WH, Graan PNE (1995) $N$-terminal-specific anti-B-50 (GAP-43) antibodies inhibit $\mathrm{Ca}^{2+}$-induced noradrenaline release, B-50 phosphorylation and dephosphorylation, and calmodulin binding. J Neurochem 64:1127-1136.

Huber G, Martin JR, Loffler J, Moreau J-L (1993) Involvement of amyloid precursor protein in memory formation in the rat: an indirect antibody approach. Brain Res 603:348-352.

Ishida A, Furukawa K, Keller JN, Mattson MP (1997) Secreted form of $\beta$-amyloid precursor protein shifts the frequency dependency for induction of LTD, and enhances LTP in hippocampal slices. NeuroReport 8:2133-2137.

Ishiguro M, Ohsawa I, Takamura C, Morimoto T, Kohsaka S (1998) Secreted form of beta-amyloid precursor protein activates protein kinase $\mathrm{C}$ and phospholipase $\mathrm{C}$ gamma 1 in cultured embryonic rat neocortical cells. Brain Res Mol Brain Res 53:24-32.

Kang J, Muller-Hill B (1990) Differential splicing of Alzheimer's disease amyloid A4 precursor RNA in rat tissues: pre A4 ${ }^{695}$ mRNA is predominantly produced in rat and human brain. Biochem Biophys Res Commun 166:1192-1200.

Kar S, Seto D, Gaudreau P, Quirion R (1996) $\beta$-amyloid-related peptides inhibit potassium-evoked acetylcholine release from rat hippocampal slices. J Neurosci 16:1034-1040.

Katz B, Miledi R (1969) Spontaneous and evoked activity of motor nerve endings in calcium ringer. J Physiol (Lond) 203:689-706.

Lewin G, Barde Y-A (1996) Physiology of the neurotrophins. Annu Rev Neurosci 19:289-317.

Lo Y-J, Poo M-M (1994) Heterosynaptic suppression of developing neuromuscular synapses in culture. J Neurosci 14:4684-4693.

Lohof AM, Lp NY, Poo M-M (1993) Potentiation of developing neuromuscular synapses by the neurotrophins NT-3 and BDNF. Nature 363:350-353.

Malinow R, Tsien RW (1990) Presynaptic enhancement shown by whole-cell recordings of long-term potentiation in hippocampal slices. Nature 346:177-180.

Mattson MP, Barger SW, Cheng B, Lieberburg I, Smith-Swintosky VL, Rydel RE (1993) $\beta$-amyloid precursor protein metabolites and loss of neuronal $\mathrm{Ca}^{2+}$ homeostasis in Alzheimer's disease. Trends Neurosci 16:409-414.

Morimoto T, Ohsawa I, Takamura C, Ishiguro M, Kohsaka S (1998a) Involvement of amyloid precursor protein in functional synapse formation in cultured hippocampal neurons. J. Neurosci Res 51:185-195.

Morimoto T, Wang XH, Poo MM (1998b) Overexpression of synaptotagmin modulates short-term synaptic plasticity at developing neuromuscular junctions. Neuroscience 82:969-978.

Morita K, Katayama Y (1984) Two types of acetylcholine receptors on the soma of primary afferent neurons. Brain Res 290:348-352.

Moya KL, Benowitz LI, Schneider GE, Allinquant B (1994) The amyloid precursor protein is developmentally regulated and correlated with synaptogenesis. Dev Biol 161:597-603.

Ninomiya H, Roch J-M, Sundsmo MP, Otero DAC, Saitoh T (1993) Amino acid sequence RERMS represents the active domain of amyloid $\beta / \mathrm{A} 4$ protein precursor that promotes fibroblast growth. J Cell Biol 121:879-886.

Ninomiya H, Roch J-M, Jin L-W, Saitoh T (1994) Secreted form of amyloid $\beta / \mathrm{A} 4$ protein precursor (APP) binds to two distinct APP binding sites on rat B103 neuron-like cells through two different domains, but only one site is involved in neuritotropic activity. J Neurochem 63:495-500.

Nitsch RM, Farber SA, Growdon JH, Wurtman RJ (1993) Release of 
amyloid $\beta$-protein precursor derivatives by electrical depolarization of rat hippocampal slices. Proc Natl Acad Sci USA 90:5191-5193.

Okado H, Okamoto H (1992) A Xenopus homologue of the human $\beta$-amyloid precursor protein: developmental regulation of its gene expression. Biochem Biophys Res Commun 189:1561-1568.

Ohsawa I, Hirose Y, Ishiguro M, Imai Y, Ishiura S, Kohsaka S (1995) Expression, purification, and neurotrophic activity of amyloid precursor protein-secreted forms produced by yeast. Biochem Biophys Res Commun 213:52-58.

Ohsawa I, Takamura C, Kohsaka S (1997) The amino-terminal region of amyloid precursor protein is responsible for neurite outgrowth in rat neocortical explant culture. Biochem Biophys Res Commun 236:59-65.

Salbaum JM, Ruddle FH (1994) Embryonic expression pattern of amyloid protein precursor suggests a role in differentiation of specific subsets of neurons. J Exp Zool 269:116-127.

Schubert W, Prior R, Weidemann A, Dircksen H, Multhaup G, Masters CL, Beyreuther K (1991) Localization of Alzheimer $\beta A 4$ amyloid precursor protein at central and peripheral synaptic sites. Brain Res 563:184-194.
Selkoe DJ (1991) The molecular pathology of Alzheimer's disease. Neuron 6:487-498.

Shapira R, Silberberg SD, Ginsburg S, Rahamimoff R (1987) Activation of protein kinase $\mathrm{C}$ augments evoked transmitter release. Nature 325:58-60.

Spitzer NC, Lamborghini JE (1976) The development of the action potential mechanism of amphibian neurons isolated in culture. Proc Natl Acad Sci USA 73:1641-1645.

Sugiyama H, Ito I, Watanabe M (1989) Glutamate receptor subtypes may be classified into two major categories: a study on Xenopus oocytes injected with rat brain mRNA. Neuron 3:129-132.

Xie Z, Poo M-M (1986) Initial events in the formation of neuromuscular synapse. Proc Natl Acad Sci USA 83:7069-7073.

Yamazaki T, Selkoe DJ, Koo EH (1995) Trafficking of cell surface $\beta$-amyloid precursor protein: retrograde and transcytotic transport in cultured neurons. J Cell Biol 129:431-442.

Yang J, Tsien RW (1993) Enhancement of N- and L-type calcium channel currents by protein kinase $\mathrm{C}$ in frog sympathetic neurons. Neuron 10:127-136. 